\title{
miR-215 suppresses papillary thyroid cancer proliferation, migration, and invasion through the AKT/GSK-3 $\beta /$ Snail signaling by targeting ARFGEF1
}

Jihua Han ${ }^{1}$, Meiyin Zhang ${ }^{2}$, Chunlei Nie', Jinliang Jia', Fengyue Wang ${ }^{3}$, Jiawei $\mathrm{Yu}^{1}$, Wen Bi, Bo Liu', Ruinan Sheng', Guoqing He${ }^{1}$, Lingyu Kong ${ }^{1}$, Lingling Zheng ${ }^{1}$, Rui Pang ${ }^{1}$, Zhaoming Ding ${ }^{1}$, Lili Chen', Qiang Guan', Shangha Pan ${ }^{3}$, Xianzhi Meng ${ }^{3}$, Jin X $\mathrm{u}^{4}$, Lianxin $\mathrm{Liu}^{3,5}$ and Jiewu Zhang ${ }^{1}$

\begin{abstract}
The incidence of papillary thyroid cancer (PTC) has been rapidly increasing in recent years. PTC is prone to lymph node metastasization, which further increases the recurrence rate and mortality of thyroid cancer. However, the underlying mechanisms of this process remain elusive. Several reports have shown that the microRNA miR-215 plays an important role in cancer metastasis. Here, we investigated, for the first time, the potential association between miR-215 and metastasis in PTC. The results of qPCR analysis demonstrated that miR-215 was downregulated in PTC cell lines and tissues, and lower levels of miR-215 correlated with lymph node metastasis of PTC. In vitro and in vivo assays revealed that restoration of miR-215 dramatically inhibited PTC cell proliferation and metastasis. We identified ADP ribosylation factor guanine nucleotide-exchange factor 1 (ARFGEF1) as the target, which mediated the function of miR-215. The expression of ARFGEF1 was inhibited by miR-215, and the effects of miR-215 were abrogated by re-expression of ARFGEF1. Moreover, we found that miR-215 suppressed PTC metastasis by modulating the epithelial-mesenchymal transition via the AKT/GSK-3 $\beta /$ Snail signaling. In summary, our study proves that miR-215 inhibits PTC proliferation and metastasis by targeting ARFGEF1 and indicates miR-215 as a biomarker for PTC prognosis.
\end{abstract}

\section{Introduction}

Thyroid cancer (TC), deriving from thyroid follicular epithelial cells or parafollicular $C$ cells, is the most frequent malignant tumor in the endocrine system. Papillary thyroid cancer (PTC) is the most common type of TC and, in recent years, its incidence has been increasing

Correspondence: Jin xu (xujinyd@aliyun.com) or Lianxin Liu

(liulx@ems.hrbmu.edu.cn) or Jiewu Zhang (drzhangjiewu@sohu.com)

${ }^{1}$ Department of Head and Neck Surgery, the Third Affiliated Hospital of Harbin

Medical University, Harbin, Heilongjiang, China

Department of Gynecology, Harbin Medical University Cancer Hospital,

Harbin, China

Full list of author information is available at the end of the article.

These authors contributed equally: Jihua Han, Meiyin Zhang, Chunlei Nie,

Jinliang Jia, Fengyue Wang

Edited by I. Amelio worldwide ${ }^{1}$. For most of the patients, the prognosis of PTC is good; however, $30 \%$ of the patients are diagnosed with lymph node metastases (LNM $)^{2}$, which increase the recurrence rate and mortality of $\mathrm{PTC}^{3}$. The knowledge of the underlying mechanisms in PTC LNM is essential to make appropriate therapeutic decisions and improve the prognosis of patients with PTC.

MicroRNAs (miRNAs) are short ( 22 nucleotides), single-stranded RNAs that regulate gene expression at the post-transcriptional level by binding to the 3 '-untranslated region ( $3^{\prime}$-UTR) of target mRNAs, leading to their degradation or inhibition of their translation ${ }^{4}$. Increasing evidence suggests that miRNAs are involved in various biological processes, including cell proliferation, migration, invasion, differentiation, and immune responses ${ }^{5}$.

\section{(c) The Author(s) 2019}

(c) Open Access This article is licensed under a Creative Commons Attribution 4.0 International License, which permits use, sharing, adaptation, distribution and reproduction c. in any medium or format, as long as you give appropriate credit to the original author(s) and the source, provide a link to the Creative Commons license, and indicate if changes were made. The images or other third party material in this article are included in the article's Creative Commons license, unless indicated otherwise in a credit line to the material. If material is not included in the article's Creative Commons license and your intended use is not permitted by statutory regulation or exceeds the permitted use, you will need to obtain permission directly from the copyright holder. To view a copy of this license, visit http://creativecommons.org/licenses/by/4.0/. 
miRNAs can act as oncogenes or tumor-suppressor genes in PTC $^{6}$. Studies have shown that miR-215 plays a critical role as a tumor suppressor in renal cell carcinoma, gastric cancer, glioma, and colorectal cancer and is a prognostic biomarker for these pathologies ${ }^{7-10}$. However, the potential effect of miR-215 in PTC metastasization has not been investigated yet.

In this study, we investigated the potential function of miR-215 in the progression and development of PTC cancer tissues, showed the downregulation of miR-215 in PTC samples, and the relationship between its aberrant expression and metastasis of PTC. Moreover, we demonstrated, in vitro and in vivo, that overexpression of miR-215 significantly suppresses tumor proliferation and metastasis of PTC by targeting the ADP ribosylation factor guanine nucleotide-exchange factor 1 (ARFGEF1). More interestingly, we also found that miR-215 can modulate the epithelial-mesenchymal transition (EMT) process through the AKT/GSK-3 $\beta /$ Snail signaling.

\section{Results}

miR-215 is downregulated in PTC tissues and cell lines

To investigate the role of miR-215 in PTC, we performed qPCR assays and measured miR-215 expression in 48 paired PTC tissues and the corresponding adjacent normal tissues (ANT). We found that miR-215 expression was significantly lower in PTC tissues than in ANT (Fig. 1a). Similarly, data from The Cancer Genome Atlas (TCGA, https://cancergenome.nih.gov/) database confirmed that miR-215 expression is downregulated in PTC tissues (Fig. 1b). Meanwhile, the survival data from the TCGA database indicated that patients with lower miR215 expression exhibited significantly poorer disease-free survival (DFS) than patients with higher miR-215 expression (Fig. 1c). Furthermore, the downregulation of miR-215 expression was negatively associated with tumor size $(P<0.05)$, differentiation $(P<0.05)$, and lymph node metastasis status $(P<0.01)$ in PTC (Supplementary Table 1). Moreover, the expression levels of miR-215 were relatively lower in four PTC cell lines (K1, BCPAP, TPC-1, and $\mathrm{IHH} 4$ ) than in the normal thyroid epithelial cell line Nthy-ori 3-1 (Fig. 1d).

Next, we silenced and overexpressed miR-215 by oligonucleotide transfection in cells, which displayed high (K1 and BCPAP) and low (TPC-1 and IHH4) miR-215 endogenous expression, respectively. $\mathrm{qPCR}$ analysis revealed that miR-215 was efficiently repressed or overexpressed with $100 \mathrm{nM}$ of inhibitor or mimic oligonucleotides in these cells, which were used for subsequent experiments (Fig. 1e).
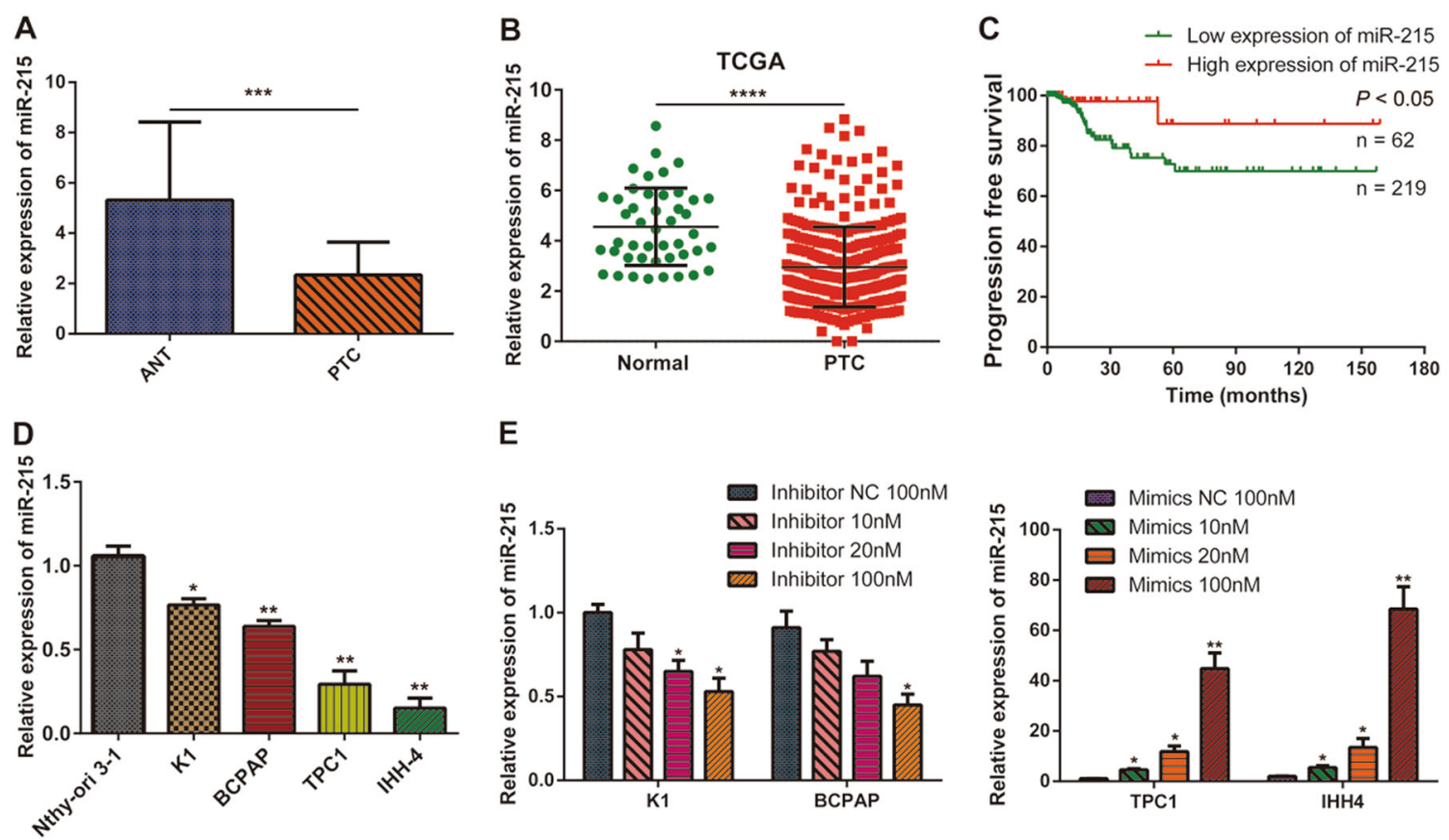

Fig. 1 miR-215 expression is decreased in PTC. a Relative miR-215 expression in PTC tissues and their corresponding ANT were detected by qPCR $(n=48)$. $\mathbf{b}$ Relative expression of miR-215 in PTC tissues and normal thyroid tissues in the TCGA database. c Kaplan-Meier analysis of PFS in PTC patients with variable expression of miR-215. d qPCR analysis of miR-215 expression in a normal thyroid epithelial cell line (Nthy-ori 3-1), and in four PTC cell lines (K1, BCPAP, TPC-1, and IHH4). e qPCR analysis of miR-215 expression after its silencing or overexpression in PTC cells. All experiments were performed in triplicate, and the results are presented as the mean $\pm \mathrm{SD}$. ${ }^{*} P<0.05$, ${ }^{* *} P<0.01,{ }^{* *} P<0.001$, and ${ }^{* * *} P<0.0001$ 
miR-215 suppresses PTC growth both in vitro and in vivo

To detect the role of miR-215 in PTC cell proliferation, we carried out CCK- 8 and colony-formation assays. The reduction of miR-215 expression significantly promoted cell growth in $\mathrm{K} 1$ and BCPAP cells, and the overexpression of miR-215 dramatically suppressed the growth of TPC-1 and IHH4 cells (Fig. 2a, b and Supplementary Figure 1).

Next, we established a xenograft model by subcutaneously injecting cells stably overexpressing miR-215 (TPC-1-Lv-miR-215) or control cells (TPC-1-Lv-NC) into the flanks of nude mice. The tumor volumes were evidently smaller in the Lv-miR-215 group than in the control group (Fig. 2c). Furthermore, IHC assays showed that the expression of $\mathrm{Ki}-67$ in the Lv-miR-215 group was lower than that in the Lv-NC group (Fig. 2d). Hence, we concluded that miR-215 inhibits PTC tumor growth both in vitro and in vivo.

\section{Ectopic expression of miR-215 inhibits PTC cell invasion in vitro and metastasis in vivo}

As mentioned above, miR-215 is related to the lymph node metastasis of PTC. Therefore, we examined the effect of miR-215 on the motility and invasiveness of PTC. Wound-healing assays indicated that miR-215 knockdown enhanced the migration of PTC cells, while miR215 overexpression suppressed cell motility compared with the control cells (Fig. 3a). Transwell migration and Matrigel invasion assays showed that the migratory and invasive capabilities of $\mathrm{K} 1$ and BCPAP cells significantly increased after miR-215 downregulation. Meanwhile, upregulation of miR-215 in TPC-1 and IHH4 cells markedly decreased their migratory and invasive behaviors (Fig. 3b and Supplementary Figure 2).

We also established a metastasis mice model by injecting stably overexpressing miR-215 (TPC-1-Lv-miR$215)$ or control cells (TPC-1-Lv-NC) into the mice tail

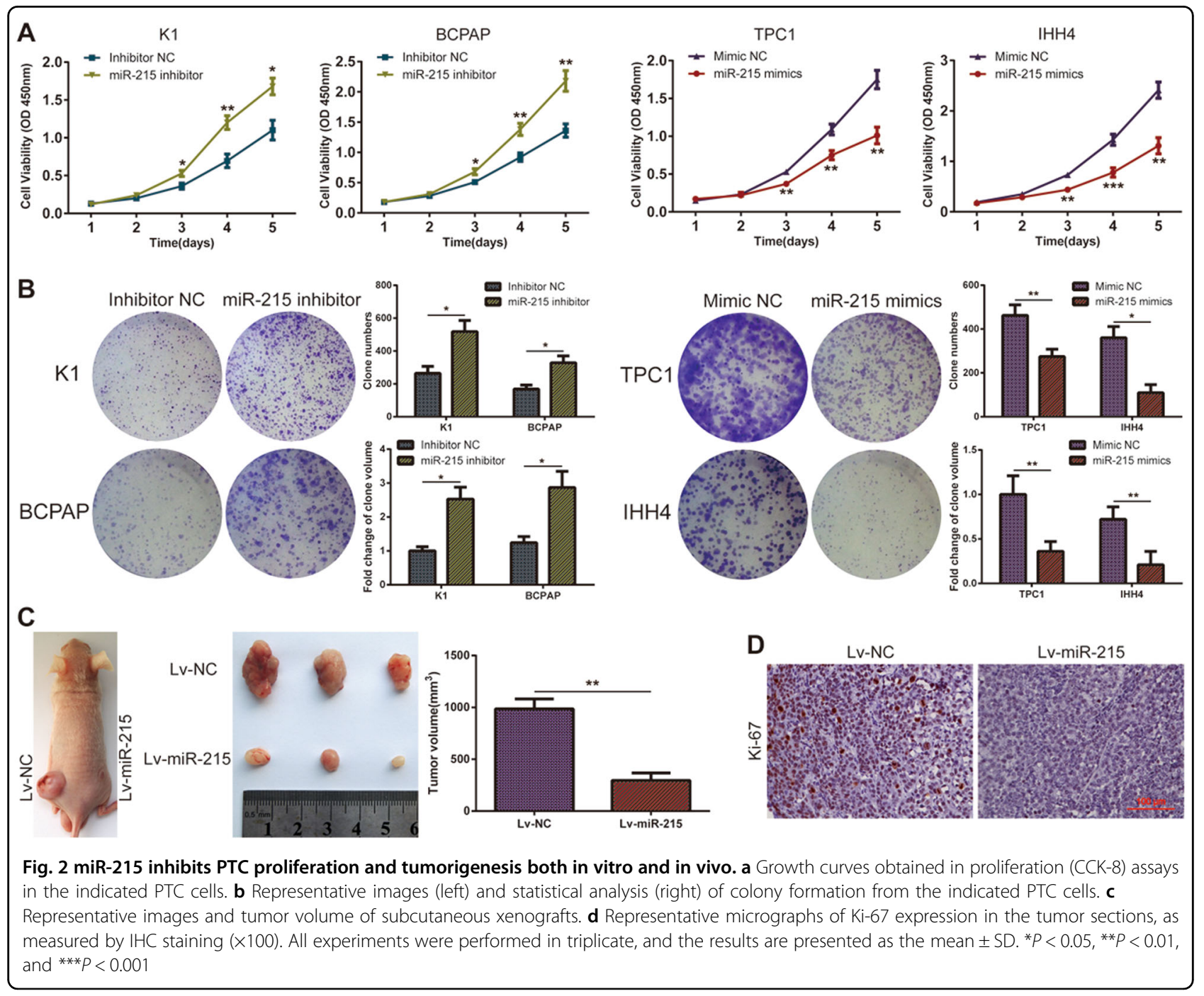


A
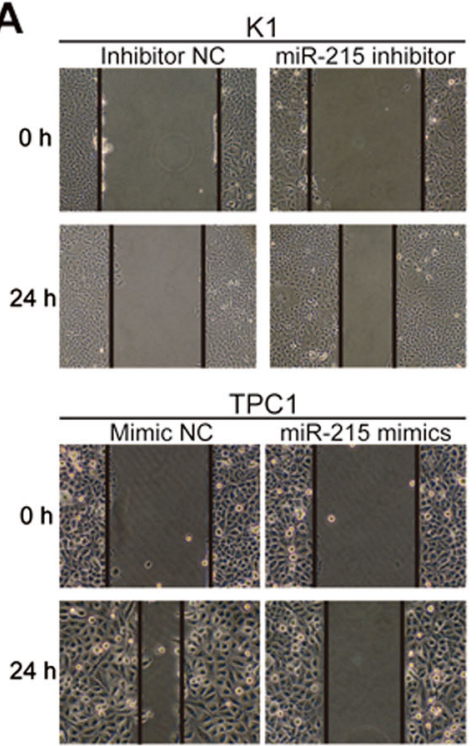

B
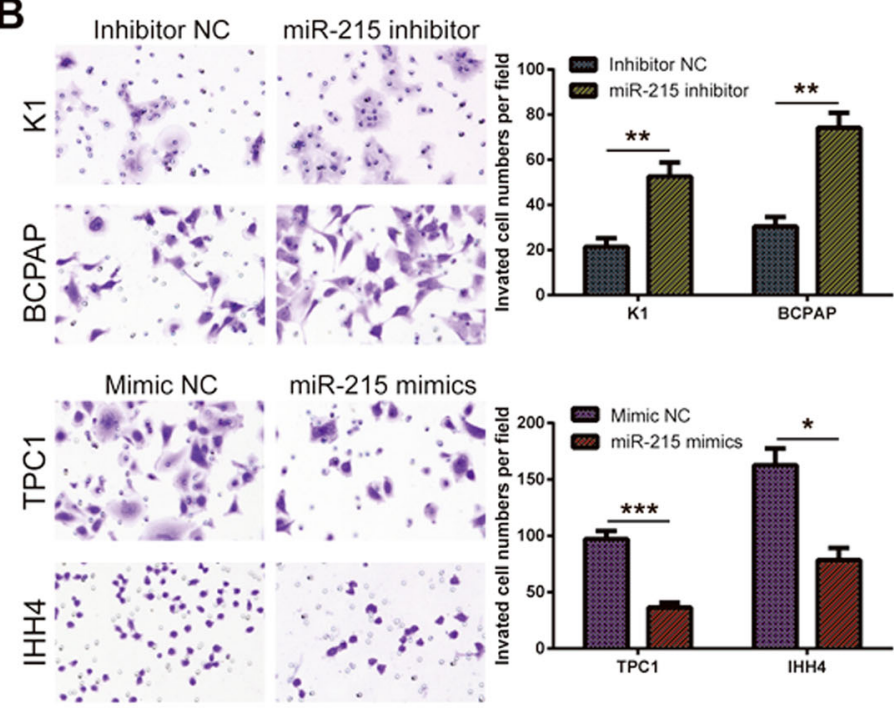

D
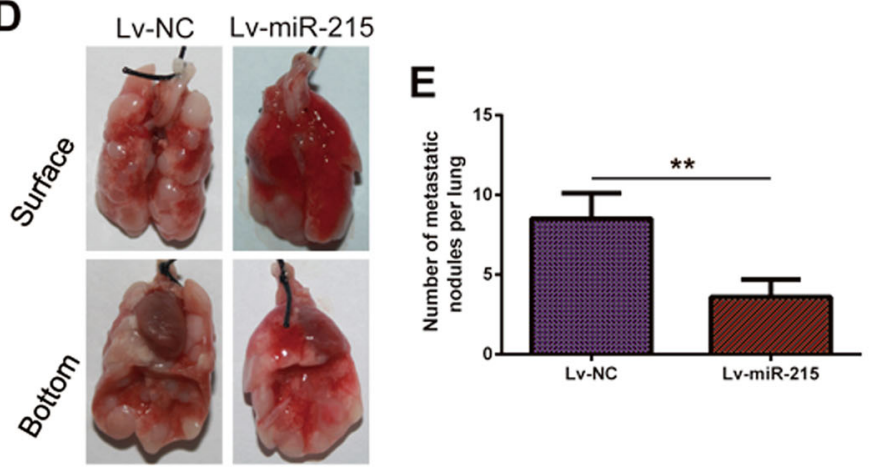
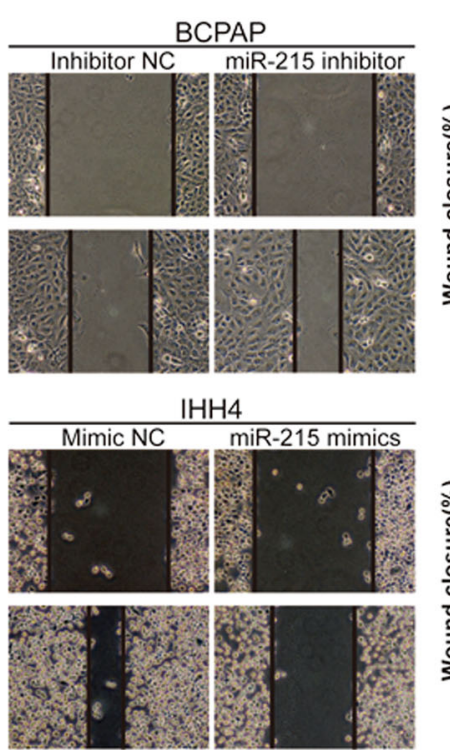

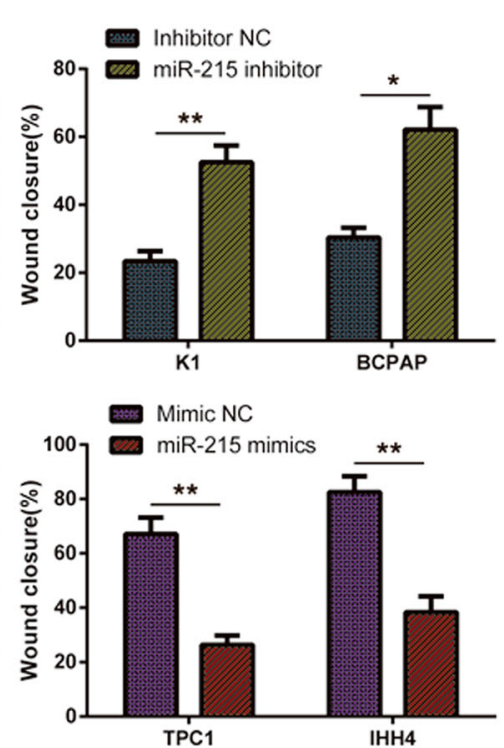

C
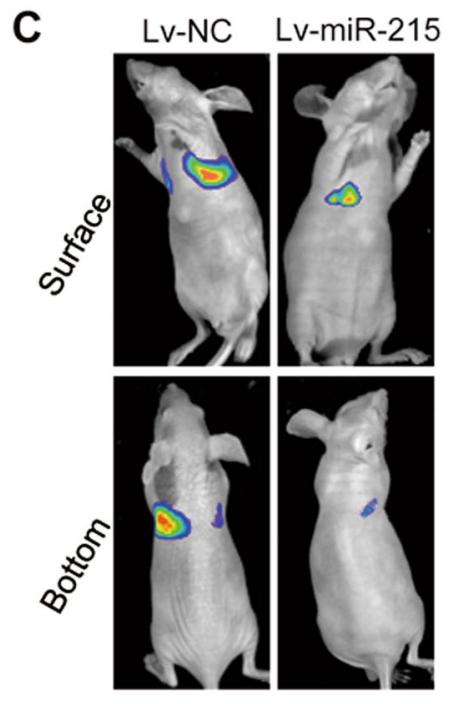

$\mathbf{F}$

Lv-NC

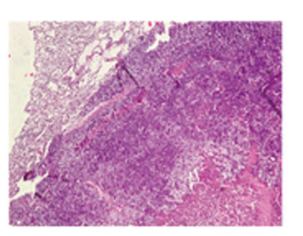

Lv-miR-215

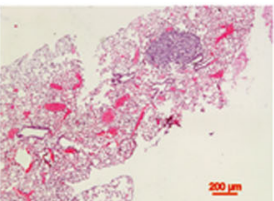

Fig. 3 miR-215 represses invasion and metastasis of PTC both in vitro and in vivo. a The migration of PTC cells, treated as indicated, was analyzed in wound-healing assays. $\mathbf{b}$ The invasive capacities of PTC cells, treated as indicated, were detected by transwell invasion assays. c Representative bioluminescence images of the lung metastasis model. $\mathbf{d}$ Representative photographs of dissected lungs from the lung metastasis model. e Number of lung metastatic nodules in the indicated groups ( $n=6$ per group). $\mathbf{f}$ Representative H\&E staining of lung metastasis samples $(\times 40)$. All experiments were performed in triplicate, and the results are presented as the mean \pm SD. ${ }^{*} P<0.05$, ${ }^{* *} P<0.01$, and ${ }^{* * *} P<0.001$ 
vein and monitoring the appearance of lung metastatic nodules. We found that less and smaller lung metastatic tumor nodules were detected in the mice injected with the miR-215 overexpressing cells compared with controls (Fig. 3c-e). Histologic analysis of the lungs isolated from the mice further confirmed the effect of miR-215 on lung metastasis (Fig. 3f). These results proved that miR-215 is an important factor for the suppression of PTC invasion and metastasis.

\section{ARFGEF1 is a direct target of miR-215}

To identify the direct downstream target through which miR-215 regulates PTC progression, we conducted bioinformatics analyses based on publicly available databases, including TargetScan (http://www.targetscan.org/ vert_72/), miRanda (http://www.microrna.org/microrna/ home.do), and miRDB (http://www.mirdb.org/miRDB/). We identified several potential candidate genes, and after reviewing of the literature, decided to focus on ARFGEF1 for further investigation.

Western blotting assays indicated that miR-215 inhibition increased the expression of ARFGEF1, while miR-215 overexpression had the opposite result (Fig. 4a). Next, we performed luciferase reporter assays to verify whether ARFGEF1 is a direct target of miR-215 (Fig. 4b and Supplementary Figure 3). These assays showed that the activity of a luciferase reporter plasmid with the wild-type 3'-UTR of ARFGEF1 upstream the luciferase coding sequence was significantly suppressed by miR-215 mimics. However, miR-215 mimics did not exert this
A

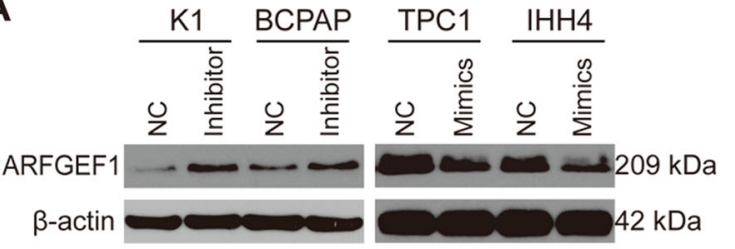

B

ARFGEF1 3'-UTR position: 1233-1240

ARFGEF1 Wt 3'-UTR $\quad 5^{\prime}$...AUUCUAAUCAGGUUAUAGGUCAA... hsa-miR-215-5p 3' CAGACAGUUAAGUAUCCAGUA

ARFGEF1 Mut 3'-UTR $55^{\prime}$... AUUCUAAUCAGGUUA ATCCAGT A... ARFGEF1 Mut' 3'-UTR 5' ... AUUCUAAUCAGGUUAUA GU AA...

D
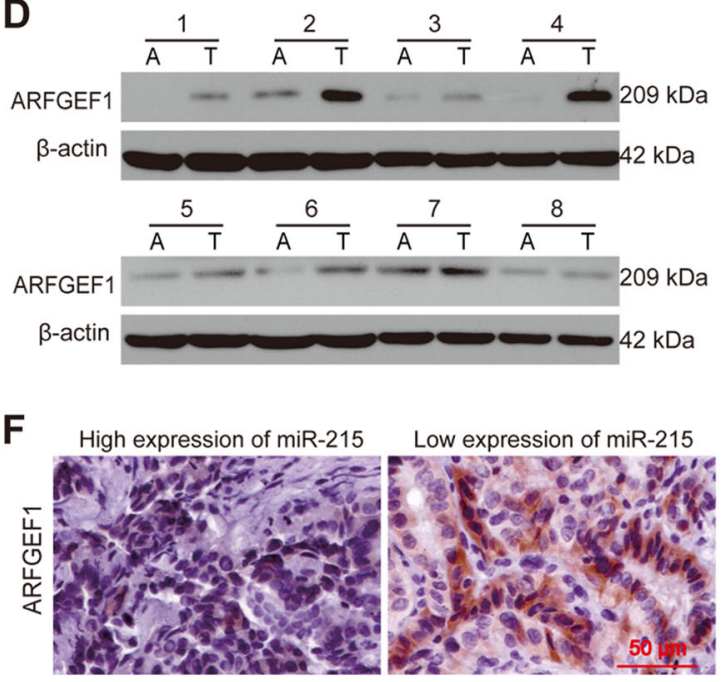

C

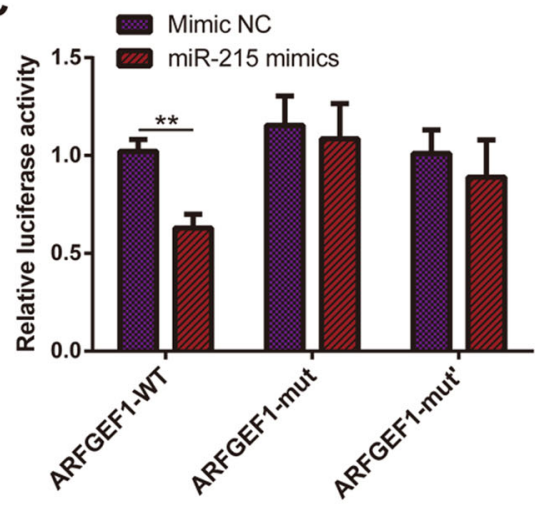

E

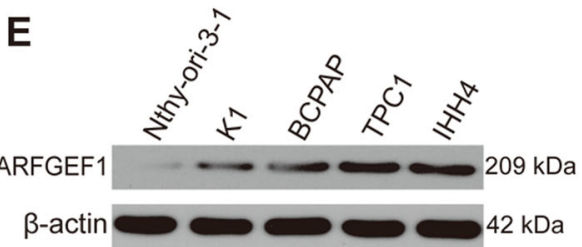

G

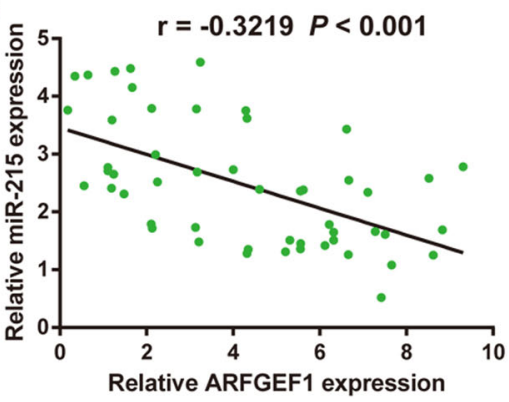

Fig. 4 ARFGEF1 is a direct downstream target of miR-215. a Western blotting analysis showing the expression of ARFGEF1 after miR-215 silencing or overexpression. b Schematic representation of miR-215 binding site in ARFGEF1 and the corresponding mutant site. $\mathbf{c}$ Luciferase assays in 293 T cells in the indicated conditions. $\mathbf{d}$ The expression of ARFGEF1 was detected in PTC tissues (T: tumor) and the corresponding normal tissues (A: adjacent normal tissues) by western blotting. e Relative expression of ARFGEF1 in Nthy-ori 3-1 and PTC cell lines. $\mathbf{f} I H C$ staining demonstrated that ARFGEF1 expression was negatively correlated with miR-215 expression $(\times 200)$. g Correlation analysis between miR-215 and ARFGEF1. All experiments were performed in triplicate, and the results are presented as the mean \pm SD. ${ }^{* *} P<0.01$ 
effect on a luciferase reporter plasmid containing $A R F$ GEF1-mutated 3'-UTR (Fig. 4c).

We also detected the expression of ARFGEF1 in human PTC tissues and cell lines by western blotting and found that ARFGEF1 was upregulated in PTC tissues and cells, as compared with the ANT and Nthy-ori 3-1, respectively (Fig. 4d, e). In addition, IHC staining indicated that ARFGEF1 expression was obviously higher in PTC tissues with low miR-215 expression than in those with high miR-215 expression (Fig. 4f). Correlation analysis revealed a negative correlation between miR-215 and ARFGEF1 $(\mathrm{r}=-0.3219, P<0.001$; Fig. $4 \mathrm{~g})$. Taken together, these data demonstrated that ARFGEF1 is a direct downstream target of miR-215 in PTC.

\section{miR-215 inhibits PTC growth and metastasis by targeting ARFGEF1}

To further investigate whether ARFGEF1 is the functional target of miR-215 in PTC, we overexpressed or silenced ARFGEF1 in BCPAP and TPC-1 cells, and investigated the subsequent effect on proliferation and metastasis. We tested two siRNAs targeting ARFGEF1. Western blotting and qPCR assays showed that siARFGEF1-2 was more efficient in knocking down ARFGEF1 in PTC cells: this siRNA was therefore chosen for further experiments (Fig. 5a and Supplementary Figure 4). Colony-formation assays revealed that silencing ARFGEF1 inhibited the proliferation of BCPAP and TPC-1 (Supplementary Figure 5). Colony-formation and CCK-8 assays indicated that the downregulation of ARFGEF1 counteracts the enhanced proliferation mediated by miR215 inhibition. On the contrary, upregulation of ARFGEF1 restores the inhibitory effect on proliferation mediated by miR-215 overexpression (Fig. $5 \mathrm{~b}$ and Supplementary Figure 6). The xenograft tumor model further confirmed these results (Fig. 5c).

Matrigel invasion and transwell migration assays revealed that the increased cell mobility associated with miR-215 inhibition was attenuated by ARFGEF1 silencing, while ARFGEF1 overexpression counteracted the effects of miR215 overexpression (Fig. 5d and Supplementary Figure 7). Furthermore, we performed experiments on the nude mice lung metastatic model to confirm these results in vivo. We found that the number of lung metastatic nodules in mice injected with TPC-1 cells was markedly decreased by miR215 overexpression and significantly increased by ARFGEF1 overexpression (Fig. 5e-g). These data suggested that miR-215 exerts its function via ARFGEF1 in PTC tumorigenicity and metastasis both in vitro and in vivo.

\section{miR-215 represses PTC metastasis by modulating EMT via the ARFGEF1/AKT/GSK-3 $\beta$ signaling}

EMT plays an important role in tumor metastasis. Therefore, we tested the potential relationship between
miR-215 and EMT. Morphology images demonstrated that treatment of BCPAP cells with a miR-215 inhibitor promotes a spindle-like (mesenchymal) morphology, whereas ARFGEF1 silencing restores a round (epithelial) morphology in BCPAP cells. On the other hand, TPC-1Lv-miR-215 cells appeared rounder than TPC-1-NC and TPC-1-Lv-miR-215-ARFGEF1 cells, overexpressing both miR-215 and ARFGEF1 (Fig. 6a).

Next, we investigated the expression of classic EMT markers by IF and IHC staining in cultured cells and xenograft tumor tissues, respectively. As expected, miR215 overexpression inhibited the expression of $\mathrm{N}$-cadherin and vimentin, whereas ARFGEF1 overexpression elevated their expression (Fig. 6b, c). These data indicated the negative association between miR-215 and EMT, and were further confirmed by western blotting assays (Fig. 6d).

Since Snail, ZEB1, ZEB2, Slug, and Twist1 were important EMT transcription factors, we took further investigation into the association between miR-215 and these transcriptional regulators. Western blotting analysis revealed that the expression of Snail was elevated or decreased on the condition that miR-215 was downregulated or overexpressed. But no noticeable alteration was found in other transcription factors, including ZEB2, which had been reported to be a direct target of miR-215 in lung cancer and pancreatic cancer ${ }^{11,12}$ (Supplementary Figure 8). In addition, the results from correlation analysis indicated that there was no obvious correlation between miR-215 and ZEB2 in PTC patients $(r=0.05866, P>0.05$; Supplementary Figure 9).

It has been reported that ARFGEF1 regulates the AKT signal pathway ${ }^{13}$. Moreover, several studies revealed that the AKT/GSK-3 $\beta /$ Snail signaling was involved in EMT. Therefore, we wondered whether miR-215 could suppress PTC metastasis by modulating EMT via the AKT signaling pathway. To validate that hypothesis, we performed western blotting assays. We found that miR-215 inhibition increases the expression of ARFGEF1, p-AKT, p-GSK-3 $\beta$, and Snail; in contrast, miR-215 overexpression decreases the levels of ARFGEF1, p-AKT, p-GSK-3 $\beta$, and Snail (Fig. 6d). However, miR-215 overexpression or inhibition did not affect the levels of the total AKT and GSK-3ß. Together, these data indicated that miR-215 inhibits PTC metastasis by repressing EMT via the ARFGEF1/AKT/ GSK-3 $\beta /$ Snail signaling (Supplementary Figure 10).

\section{Discussion}

The incidence of PTC is steadily increasing, and recurrence and metastasis hinder its favorable prognosis after treatment ${ }^{14}$. Many miRNAs have been proved to serve as biomarkers for disease diagnosis and prognosis in malignant tumors, including PTC ${ }^{15,16}$. However, the role of miR-215 in PTC remains undefined. In the present study, we show that miR-215 inhibits tumorigenesis and 


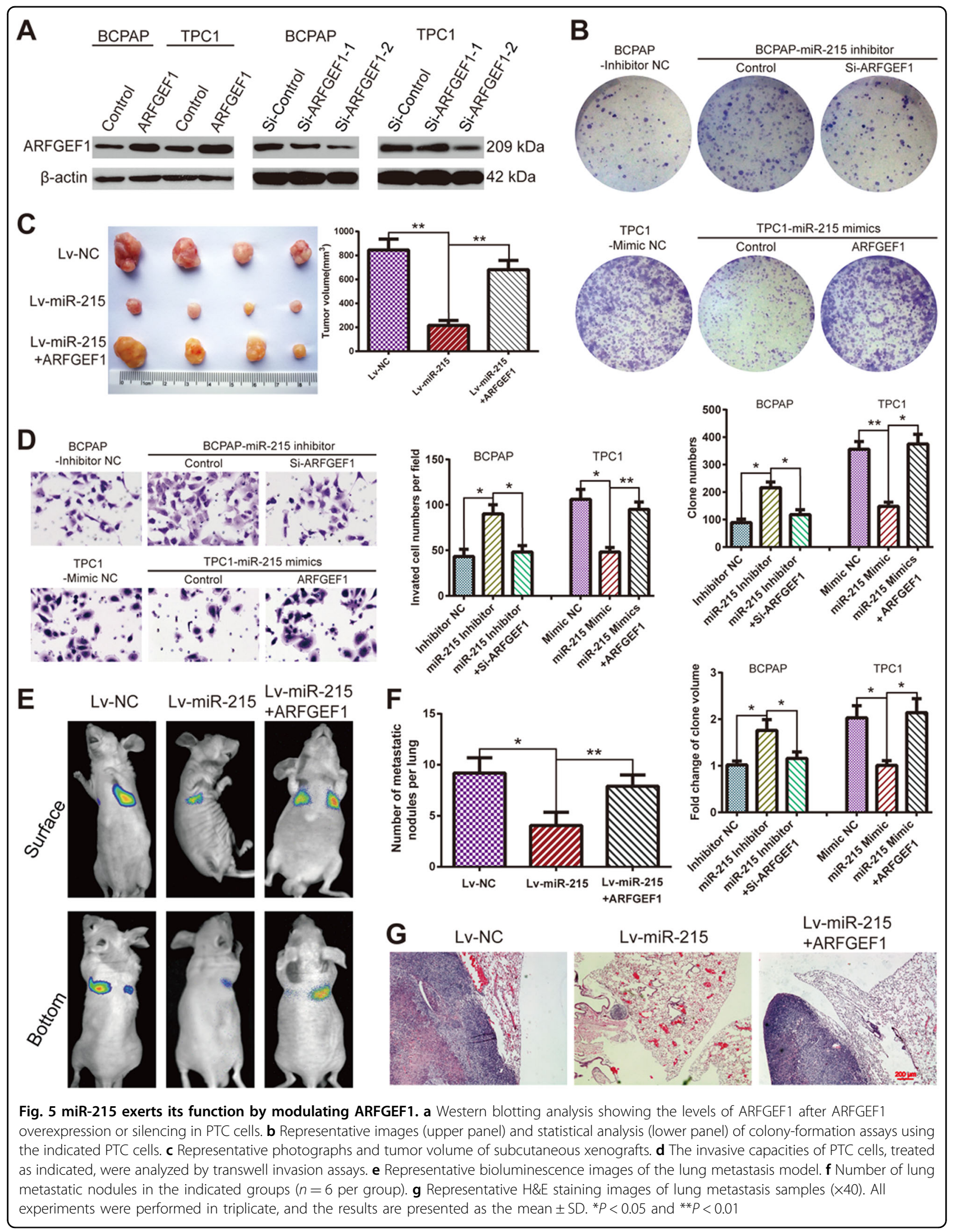



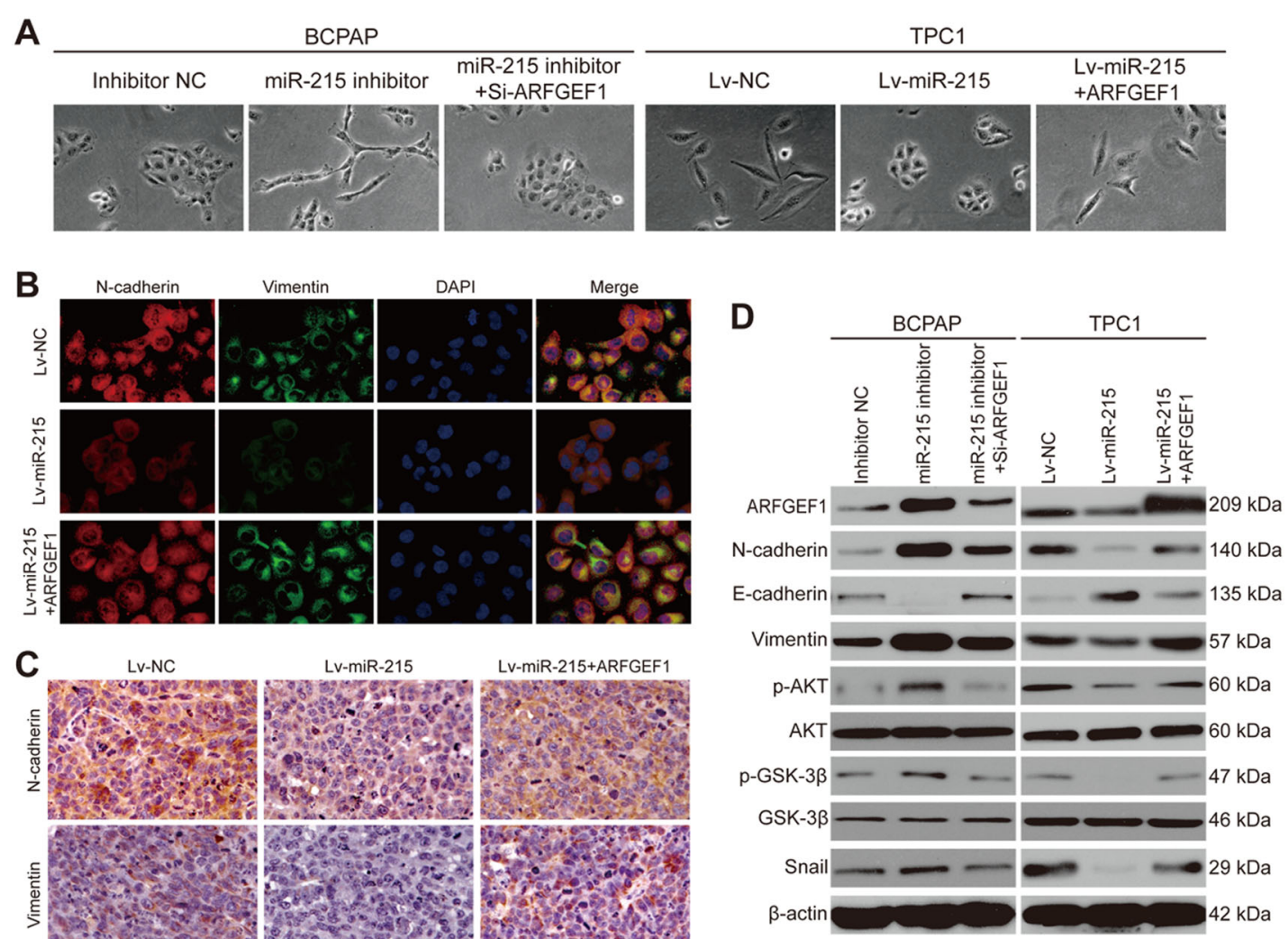

Fig. 6 miR-215 inhibits EMT in PTC by modulating the ARFGEF1/AKT/GSK-3ß/Snail pathway. a Representative morphology images of the indicated PTC cells. b Representative IF staining images relative to N-cadherin and vimentin expression in the indicated PTC cells ( $\times 200)$. c Representative $\mathrm{IHC}$ images relative to $\mathrm{N}$-cadherin and vimentin expression in the indicated tumor sections $(\times 200)$. $\mathbf{d}$ Western blotting analysis of protein expression in the indicated PTC cells

metastasis of PTC through the regulation of the AKT/ GSK-3 $\beta /$ Snail signaling by targeting ARFGEF1.

Previous studies have shown that the deregulation of miR-215 exerts an important role in some cancers. Georges et al. demonstrated that miR-215 can inhibit colon cancer proliferation by regulating the p53 network $^{17}$. In addition, miR-215 has been reported as a tumor suppressor in osteosarcoma, breast cancer, and intestinal tumor organoids ${ }^{18-21}$. On the contrary, several other studies have described miR-215 as an oncogenepromoting malignant progression in pancreatic carcinomas, gastric cancer, and glioma ${ }^{22-25}$. Here, we proved that miR-215 was significantly downregulated in PTC tissues and cell lines; our data also indicated that miR-215 downregulation is associated with PTC tumor size, metastasis status, and PFS.

We conducted gain- and loss-of-function studies to reveal the role of miR-215 in PTC. We found that miR215 inhibits proliferation, as validated by CCK- 8 and colony-formation assays, and further verified these data in a subcutaneous xenograft model. Meanwhile, miR-215 inhibits the metastasis of PTC, as demonstrated in wound-healing and transwell assays, and in an in vivo lung metastasis model.

It is well recognized that miRNAs exert their function by post transcriptionally modulating their mRNA target ${ }^{26}$. Therefore, we attempted identifying the miR-215 target gene by using publicly available databases. Among the candidates, ARFGEF1 was screened for further studies. We showed that miR-215 inhibits ARFGEF1 endogenous expression. Dual luciferase assays proved that miR-215 directly targets $A R F G E F 1$ by complementarily binding to its 3 '-UTR. Rescue experiments indicated that ARFGEF1 knockdown partly mimicked the inhibitory function of miR-215, while ARFGEF1 overexpression attenuated the effects of miR-215 overexpression, demonstrating that ARFGEF1 mediates the activity of miR-215.

EMT plays an important role in tumor metastasis ${ }^{27}$. We checked the expression of classic markers of EMT, and as expected, we found that miR-215 overexpression or ARFGEF1 knockdown inhibited EMT, whereas, miR-215 silencing and ARFGEF1 ectopic expression promoted it. Several EMT key transcriptional factors, including Snail and ZEB2, displayed a significant role in modulating the 
EMT process, among which, Snail was detected to be distinctively suppressed by miR-215 in PTC. Despite the fact that ZEB2 was confirmed as a direct target of miR215 in several other studies ${ }^{11,12,28}$, we found that there was no significant correlation between miR-215 and ZEB2 in PTC.

ARFGEF1, also called Brefeldin A-inhibited guanine nucleotide-exchange factor 1 (BIG1), is a high-molecularweight ADP-ribosylation-factor-specific guanine nucleotideexchange factor (ARF-GEF) ${ }^{29,30}$. It has been reported that BIG1 plays a critical role in neurite development ${ }^{13}$, initiation of myelination ${ }^{31}$, and membrane trafficking, and contributes to cell adhesion and migration ${ }^{32-34}$. Recently, Matsuyama et al. have revealed that ARFGEF1 is a direct target of miR$27 \mathrm{~b}$, and promotes cell proliferation in colon cancer by activating the Akt pathway ${ }^{35}$.

Meanwhile, the AKT/GSK-3 $\beta /$ Snail axis is important in modulating $\mathrm{EMT}^{36,37}$. We found that miR-215 can inhibit AKT phosphorylation mediated by ARFGEF1, and subsequently impair GSK-3 $\beta$ phosphorylation and Snail expression. Thereby, we demonstrated that miR-215 inhibits EMT via the ARFGEF1-activated AKT/GSK-3 $\beta /$ Snail signaling.

In conclusion, we demonstrated that miR-215 was downregulated in PTC tissues and cell lines, and was negatively associated with prognosis in patients with PTC. Moreover, miR-215 inhibited proliferation and metastasis in vitro and in vivo by suppressing ARFGEF1 and inhibiting the AKT/GSK-3 $\beta /$ Snail signaling pathway. Finally, our research suggests that miR-215 may be a novel prognostic marker for PTC.

\section{Materials and methods Patient samples}

Forty-eight pairs of human PTC and ANT were collected from the Department of Head and Neck Surgery, The Third Affiliated Hospital of Harbin Medical University (China) from May 2016 to August 2017. PTC samples were classified based on World Health Organization criteria. Informed consent was obtained by all patients whose biological samples were used in the study, and the experimental protocol for human subjects was approved by the Research Ethics Committee of the Third Affiliated Hospital of Harbin Medical University.

\section{Quantitative PCR (qPCR)}

The total RNA of tissues and cultured cells was extracted with the RNAeasy Mini Kit (Qiagen, Valencia, CA, USA) according to the manufacturer's instructions, and reverse-transcribed with the TaqMan MicroRNA Reverse Transcription Kit or High Capacity Reverse Transcription Kit (both from Applied Biosystems, Foster City, CA, USA). qPCR assays were performed in duplicate with TaqMan Universal PCR Master Mix II no UNG
(Applied Biosystems) or GoTaq qPCR Master Mix (Promega, Madison, WI, USA) on the ABI7900HT Real-Time PCR system (Applied Biosystems). Data were analyzed using the $2^{-\Delta \Delta C t}$ method. The miRNA and mRNA expression levels were normalized to U6 and glyceraldehyde 3 phosphate dehydrogenase (GAPDH), respectively. The probes for U6 and miR-215 (Cat\#: 4426961) were purchased from Thermofisher Scientific (San Jose, CA, USA). The sequence of primers used for qPCR were ARFGEF1 Fwd: 5'-CGAACATCAAAGTCCACC-3' and Rev: 5'-ACAGACCACAAGCACCAC-3'; ZEB2 Fwd: 5'TTCCTGGGCTACGACCATACC-3' and Rev: $5^{\prime}$-CAAG CAATTCTCCCTGAAATCC-3'; GAPDH Fwd: 5'-GCA CCGTCAAGGCTGAGAAC- $3^{\prime}$ and Rev: $5^{\prime}$-TGGTGAAG ACGCCAGTGGA-3'.

\section{Cell culture}

The human normal thyroid epithelial cell line Nthy-ori 3-1 was purchased from the European Collection of Cell Culture (ECACC, Salisbury, UK). PTC cell lines (TPC-1, $\mathrm{K} 1$, BCPAP, and IHH4) were purchased from Cell Bank of Type Culture Collection of the Chinese Academy of Sciences, Shanghai Institute of Cell Biology (Shanghai, China). TPC-1, BCPAP, and IHH4 cells were cultured in Roswell Park Memorial Institute medium (RPMI) 1640 supplemented with $10 \%$ fetal bovine serum (FBS), Nthy-ori 3-1 cells were cultured in F12K medium supplemented with $10 \%$ FBS, and K1 cells were cultured in Dulbecco's modified Eagle's medium (DMEM) supplemented with $10 \% \mathrm{FBS}$. All the cells were incubated in a $5 \%$ $\mathrm{CO}_{2}$ incubator at $37^{\circ} \mathrm{C}$.

\section{Lentivirus and reagents}

miR-215 mimics, miR-215 inhibitor, and their corresponding negative control (NC) oligonucleotide were synthesized by RiboBio Co. Ltd. (Guangzhou, China). Small interfering RNA against ARFGEF1 (si-ARFGEF1) and nonspecific NC siRNA were purchased from GenePharma Co., Ltd. (Shanghai). Lentiviral vectors for human miR-215 and ARFGEF1 overexpression (Lv-miR-215 and Lv-ARFGEF1) or the plasmids for luciferase reporter study were purchased from GeneChem Co. Ltd. (Shanghai). After the cells were infected with lentiviral vectors, we selected the cells for 2 weeks with $1 \mu \mathrm{g} / \mathrm{mL}$ puromycin (Sigma-Aldrich, St. Louis, MO, USA).

\section{Cell counting kit-8 (CCK8) and colony-formation assays}

Cell proliferation was assessed using the CCK8 kit (Dojindo, Kumamoto, Japan). The cells were seeded in a 96-well plate at a density of 2,000-4,000 per well and their viability was determined $24-72 \mathrm{~h}$ later. The cell medium was replaced with $100 \mu \mathrm{L}$ of complete medium supplemented with $10 \mu \mathrm{L}$ of CCK8, and the cells were incubated at $37^{\circ} \mathrm{C}$ with $5 \% \mathrm{CO}_{2}$ for $2 \mathrm{~h}$. Absorbance values (OD) 
were measured at $450 \mathrm{~nm}$ in a microplate reader (SpectraMax M2, Molecular Devices, CA, USA).

Colony-formation assays were performed using 0.5\% crystal violet, after the cells were plated in six-well plates at a density of 500 cells per well and cultured at $37^{\circ} \mathrm{C}$ with $5 \% \mathrm{CO}_{2}$ for 14 days. Images were captured with a Nikon microscope (Tokyo, Japan).

\section{Wound-healing assays}

Cells were seeded in six-well plates and allowed to grow to confluence. The monolayer was scratched with a micropipette tip, the cells were washed three times in a medium, and images of the wound area were captured at 0 and $24 \mathrm{~h}$ with an Eclipse microscope (Nikon).

\section{Transwell migration and invasion assays}

Transwell chambers (Corning, NY, USA) were used to perform transwell migration assays. Cells were seeded in serum-free media into the upper chamber and maintained at $37^{\circ} \mathrm{C}$. The bottom of the chamber was filled with normal media. Matrigel (BD Biosciences, Franklin Lakes, NJ, USA) was used to pre-coat the chamber membrane in 24-well dishes for the invasion assays. The chambers were maintained in a $5 \% \mathrm{CO}_{2}$ incubator at $37^{\circ} \mathrm{C}$ for $24-48 \mathrm{~h}$. The cells on the lower side of the filter were then stained with $0.5 \%$ crystal violet and counted under a light microscope (Olympus, Tokyo, Japan, $\times 200$ magnification).

\section{Western blot analysis}

Total proteins from the cells or tissues were extracted using a protein lysis buffer with PhosStop phosphatase inhibitor cocktail and protease inhibitor cocktail (both from Roche Diagnostics, Indianapolis, IN, USA). Lysates were denatured prior to sodium dodecyl sulfate polyacrylamide gel electrophoresis (SDS-PAGE) and then transferred to polyvinylidene difluoride (PVDF) membranes (Millipore, Hertfordshire, UK). The membranes were blocked with $5 \%$ nonfat milk at $20-25^{\circ} \mathrm{C}$ for $1 \mathrm{~h}$ and then incubated with primary antibodies overnight at $4{ }^{\circ} \mathrm{C}$. The following day, the membranes were washed and further incubated with secondary antibodies for $1 \mathrm{~h}$. The immunoreactive signals were visualized using enhanced chemiluminescence reagents (ECL, Pierce, Rockford, USA). The following antibodies were used: anti-ARFGEF1 (Abcam, ab183747, Cambridge, MA, USA), anti-Ki-67 (Abcam, ab15580), anti-Vimentin (Cell Signaling, \#3932, Beverly, MA, USA), anti-E-cadherin (Cell Signaling, \#3195), anti-N-Cadherin (Cell Signaling, \#13116), antiSnail (Cell Signaling, \#3879), anti-Slug (Cell Signaling, \#9585), anti-ZEB1 (Cell Signaling, \#3396), anti-Twist1 (Cell Signaling, \#46702), anti-ZEB2 (Abcam, ab138222), anti-AKT (Cell Signaling, \#4685), anti-p-AKT (Cell Signaling, \#4060), anti-GSK-3 $\beta$ (Abcam, ab32391), anti-p-
GSK-3 $\beta$ (Abcam, ab75745), and anti- $\beta$-actin (Sigma, A5441).

\section{Immunohistochemistry (IHC) assays}

Tissues were fixed with $10 \%$ formalin and embedded in paraffin. Tissue sections were deparaffinized in xylene, rehydrated in a serial of graded ethanol, and antigens were retrieved by boiling in an antigen unmasking solution (Vector lab, Burlingame, CA, USA). Endogenous peroxidase activity was blocked with $10 \%$ goat serum for 30 min, after which the tissue sections were incubated with a primary antibody at $4{ }^{\circ} \mathrm{C}$ overnight, and then with a secondary antibody (Vector Lab) for $1 \mathrm{~h}$. The immunocomplexes were visualized with a 3,3'-diaminobenzidine tetrahydrochloride solution (DAB kit, Vector lab) and the sections were stained with hematoxylin (Sigma). Slides were dehydrated through ethanol, sealed with coverslips, and imaged under a light microscope (Olympus, $\times 200$ magnification).

\section{Luciferase reporter assays}

Cells were seeded in 24-well plates and co-transfected with the plasmids containing the wild-type or mutated 3 'UTR sequence of ARFGEF1, pRL-TK Renilla, and miR215 mimics using Lipofectamine 3000 (Invitrogen). The luciferase activity was measured after incubation for $48 \mathrm{~h}$ with the Dual Luciferase Reporter Assay System (Promega) according to the manufacturer's instructions.

\section{Immunofluorescence (IF) assays}

PTC cells were fixed with $4 \%$ paraformaldehyde, permeabilized with $0.5 \%$ Triton X-100 (Sigma), blocked by $10 \%$ normal goat serum (Vector Lab), and incubated with primary antibodies overnight at $4{ }^{\circ} \mathrm{C}$. Then, the cells were incubated with Alexa Fluor 488- or 555-conjugated secondary antibodies (Invitrogen), stained with DAPI (Vector $\mathrm{Lab}$ ), and photographed under a fluorescence microscope (Olympus).

\section{Xenograft nude mouse model}

All-male BALB/c athymic nude mice (4-6 weeks old) were purchased from the Experimental Animal Center of Shanghai Institute for Biological Sciences (China) and maintained in pathogen-free conditions. The procedures were performed in accordance with the institutional guidelines for animal care, and approved by the Committee on the Use of Live Animals in Teaching and Research of the Harbin Medical University.

To establish the subcutaneous xenograft model, $\sim 5 \times$ $10^{6}$ tumor cells in $0.1 \mathrm{~mL}$ of phosphate-buffered saline were injected subcutaneously into the flanks of the nude mice. Four weeks later, the animals were euthanized, and the tumors were excised and weighed. The tumor volume 
was calculated using the equation: volume $=$ width $^{2} \times$ length $\times 0.5\left(\mathrm{~mm}^{3}\right)$.

For the in vivo metastasis assays, $5 \times 10^{6}$ cells were injected into the lateral tail vein of nude mice. The mice were imaged after 6 weeks using a NightOWL LB983 bioluminescence system (Berthold Technologies, Wildbad, Germany) upon intraperitoneal injection of D-luciferin (Promega) under anesthesia. Eight weeks later, the mice were killed, and the lungs were resected and fixed in $10 \%$ formalin for hematoxylin and eosin (H\&E) staining.

\section{Statistical analysis}

The results are presented as mean values \pm standard deviation (SD) of at least three independent experiments and were analyzed with SPSS 20.0 software (SPSS, Chicago, IL, USA) or Prism 6.0 software (GraphPad, San Diego, CA, USA). Statistical significance between two groups was analyzed using the Student's $t$ test, while multiple-group comparison was performed with one-way analysis of variance (ANOVA). P-values less than 0.05 were considered statistically significant.

\section{Acknowledgements}

This study was supported by National Key Program for Science and Technology Research and Development (Grant nos. 2016YFC0106503, 2016YFC0106500, and 2016YFC0905902), the National Natural Scientific Foundation of China (Grant no. 81602058), Postdoctoral Research Foundation of China (Grant no. 2018M630368), Hei Longjiang Postdoctoral Foundation (Grant nos. LBH-Z17143 and LBH-Q17122), Haiyan Research Fund of the Third Affiliated Hospital of Harbin Medical University (Grant nos. JJZD2017-03 and JJQN2018-14), the Fundamental Research Funds for the Provincial Universities (Grant no. 2017LCZX24), the Foundation of the First Affiliated Hospital of Harbin Medical University (Grant no. 2018B017), and The Youth Talent Support Program of Harbin Medical University Cancer Hospital (Grant no. BJQN201801). The funders had no role in the study design, data collection and analysis, decision to publish, or preparation of the paper.

\section{Author details}

${ }^{1}$ Department of Head and Neck Surgery, the Third Affiliated Hospital of Harbin Medical University, Harbin, Heilongjiang, China. ${ }^{2}$ Department of Gynecology, Harbin Medical University Cancer Hospital, Harbin, China. ${ }^{3}$ Key Laboratory of Hepatosplenic Surgery, Ministry of Education, Department of General Surgery, the First Affiliated Hospital of Harbin Medical University, Harbin, Heilongjiang, China. ${ }^{4}$ Department of Cell Biology, Harbin Medical University, Harbin, China. ${ }^{5}$ Department of Hepatobiliary Surgery, the First Affiliated Hospital of USTC, Division of Life Sciences and Medicine, University of Science and Technology of China, Hefei, Anhui, China

\section{Conflict of interest}

The authors declare that they have no conflict of interest.

\section{Publisher's note}

Springer Nature remains neutral with regard to jurisdictional claims in published maps and institutional affiliations.

Supplementary Information accompanies this paper at (https://doi.org/ 10.1038/s41419-019-1444-1).

Received: 24 October 2018 Revised: 29 January 2019 Accepted: 14 February 2019

Published online: 27 February 2019

\section{References}

1. Sturgeon, C., Yang, A. \& Elaraj, D. Surgical management of lymph node compartments in papillary thyroid cancer. Surg. Oncol. Clin. N. Am. 25, 17-40 (2016).

2. Wu, M. H., Shen, W. T., Gosnell, J. \& Duh, Q. Y. Prognostic significance of extranodal extension of regional lymph node metastasis in papillary thyroid cancer. Head \& Neck. 37, 1336-1343 (2015).

3. Amit, M. et al. Association of lymph node density with survival of patients with papillary thyroid cancer. JAMA Otolaryngol. \& Neck Surg. 144, 108-114 (2017).

4. Eulalio, A., Huntzinger, E. \& Izaurralde, E. Getting to the root of miRNAmediated gene silencing. Cell 132, 9-14 (2008).

5. Bracken, C. P., Scott, H. S. \& Goodall, G. J. A network-biology perspective of microRNA function and dysfunction in cancer. Nat. Rev. Genet. 17, 719-732 (2016).

6. Aragon Han, P. et al. MicroRNA expression and association with clinicopathologic features in papillary thyroid cancer: a systematic review. Thyroid: Off. J. Am. Thyroid. Assoc. 25, 1322-1329 (2015).

7. Deng, Y. et al. MiR-215 modulates gastric cancer cell proliferation by targeting RB1. Cancer Lett. 342, 27-35 (2014).

8. $\mathrm{Hu}$, J. et al. MiR-215 is induced post-transcriptionally via HIF-Drosha complex and mediates glioma-initiating cell adaptation to hypoxia by targeting KDM1B. Cancer Cell. 29, 49-60 (2016).

9. White, N. M. et al. miRNA profiling in metastatic renal cell carcinoma reveals a tumour-suppressor effect for miR-215. Br. J. Cancer 105, 1741-1749 (2011).

10. Vychytilova-Faltejskova, P. et al. MiR-215-5p is a tumor suppressor in colorectal cancer targeting EGFR ligand epiregulin and its transcriptional inducer HOXB9. Oncogenesis 6, 399 (2017).

11. Li, Q. W. et al. MicroRNA-215 functions as a tumor suppressor and directly targets ZEB2 in human pancreatic cancer. Genet. Mol. Res.: Gmr. 14, 16133-16145 (2015)

12. Hou, Y. et al. miR-215 functions as a tumor suppressor and directly targets ZEB2 in human non-small cell lung cancer. Oncol. Lett. 10, 1985-1992 (2015).

13. Zhou, C. et al. BIG1, a brefeldin A-inhibited guanine nucleotide-exchange protein regulates neurite development via PI3K-AKT and ERK signaling pathways. Neuroscience 254, 361-368 (2013).

14. Bates, M. F. et al. Back so soon? Is early recurrence of papillary thyroid cancer really just persistent disease? Surgery 163, 118-123 (2018).

15. Ganju, A. et al. miRNA nanotherapeutics for cancer. Drug Discov. Today 22 424-432 (2017).

16. Stokowy, T., Gawel, D. \& Wojtas, B. Differences in miRNA and mRNA Profile of Papillary Thyroid Cancer Variants. Int. J. Endocrinol. 2016, 1427042 (2016).

17. Georges, S. A. et al. Coordinated regulation of cell cycle transcripts by p53Inducible microRNAs, miR-192 and miR-215. Cancer Res. 68, 10105-10112 (2008).

18. Song, B. et al. Molecular mechanism of chemoresistance by miR-215 in osteosarcoma and colon cancer cells. Mol. Cancer 9, 96 (2010).

19. Khella, H. W. et al. miR-192, miR-194 and miR-215: a convergent microRNA network suppressing tumor progression in renal cell carcinoma. Carcinogenesis 34, 2231-2239 (2013).

20. Zhou, S. W. et al. Aberrant miR-215 expression is associated with clinical outcome in breast cancer patients. Med. Oncol. 31, 259 (2014).

21. Nakaoka, T. et al. Cluster microRNAs miR-194 and miR-215 suppress the tumorigenicity of intestinal tumor organoids. Cancer Sci. 108, 678-684 (2017).

22. Choi, D. H., Park, S. J. \& Kim, H. K. miR-215 overexpression distinguishes ampullary carcinomas from pancreatic carcinomas. Hepatobiliary \& Pancreat. Dis. Int.: HBPD Int. 14, 325-329 (2015).

23. Chen, Z., Liu, K., Li, L., Chen, Y. \& Du, S. miR-215 promotes cell migration and invasion of gastric cancer by targeting Retinoblastoma tumor suppressor gene 1. Pathol. Res. Pract. 213, 889-894 (2017).

24. Zang, Y., Wang, T., Pan, J. \& Gao, F. miR-215 promotes cell migration and invasion of gastric cancer cell lines by targeting FOXO1. Neoplasma 64, 579-587 (2017).

25. Meng, X. \& Shi, B. miR-215 functions as an oncogene in high-grade glioma by regulating retinoblastoma 1. Biotechnol. Lett. 39, 1351-1358 (2017).

26. Romano, G. \& Kwong, L. N. Diagnostic and therapeutic applications of miRNAbased strategies to cancer immunotherapy. Cancer Metastas-. Rev. 37, 45-53 (2018).

27. Karlsson, M. C., Gonzalez, S. F., Welin, J. \& Fuxe, J. Epithelial-mesenchymal transition in cancer metastasis through the lymphatic system. Mol. Oncol. 11, 781-791 (2017). 
28. Chen, D. L. et al. Long non-coding RNA UICLM promotes colorectal cancer liver metastasis by acting as a ceRNA for microRNA-215 to regulate ZEB2 expression. Theranostics 7, 4836-4849 (2017).

29. Yamaji, R. et al. Identification and localization of two brefeldin Ainhibited guanine nucleotide-exchange proteins for ADP-ribosylation factors in a macromolecular complex. Proc. Natl. Acad. Sci. USA 97 2567-2572 (2000).

30. Zhao, X., Lasell, T. K. \& Melancon, P. Localization of large ADP-ribosylation factor-guanine nucleotide exchange factors to different Golgi compartments: evidence for distinct functions in protein traffic. Mol. Biol. Cell. 13, 119-133 (2002).

31. Miyamoto, Y. et al. BIG1/Arfgef1 and Arf1 regulate the initiation of myelination by Schwann cells in mice. Sci. Adv. 4, eaar4471 (2018).

32. Li, C. C., Le, K., Kato, J., Moss, J. \& Vaughan, M. Enhancement of beta-catenin activity by BIG1 plus BIG2 via Arf activation and CAMP signals. Proc. Natl. Acad. Sci. USA 113, 5946-5951 (2016).
33. Li, C. C. et al. Effects of brefeldin A-inhibited guanine nucleotide-exchange (BIG) 1 and KANK1 proteins on cell polarity and directed migration during wound healing. Proc. Natl. Acad. Sci. USA 108, 19228-19233 (2011).

34. Le, K., Li, C. C., Ye, G., Moss, J. \& Vaughan, M. Arf guanine nucleotide-exchange factors $\mathrm{BIG} 1$ and $\mathrm{BIG} 2$ regulate nonmuscle myosin $\| \mathrm{A}$ activity by anchoring myosin phosphatase complex. Proc. Natl. Acad. Sci. USA 110, E3162-E3170 (2013).

35. Matsuyama, R., Okuzaki, D., Okada, M. \& Oneyama, C. MicroRNA-27b suppresses tumor progression by regulating ARFGEF1 and focal adhesion signaling. Cancer Sci. 107, 28-35 (2016).

36. Maseki, S. et al. Acquisition of EMT phenotype in the gefitinib-resistant cells of a head and neck squamous cell carcinoma cell line through Akt/GSK-3beta/ snail signalling pathway. Br. J. Cancer 106, 1196-1204 (2012).

37. Meng, Q. et al. Abrogation of glutathione peroxidase-1 drives EMT and chemoresistance in pancreatic cancer by activating ROS-mediated Akt/GSK3beta/ Snail signaling. Oncogene 37, 5843 (2018). 